Available online on 15.02.2020 at http://jddtonline.info
Open Access to Pharmaceutical and Medical Research
unrestricted non-commercial use, provided the original work is properly cited

Open Access

Research Article

\title{
Antimicrobial and Antioxidant Activities of Flavonoids Extracted from Pistacia lentiscus $L_{\text {., }}$ Leaves
}

\author{
Bakli Sabrina 1,2*, Harzallah Daoud 1, Zerroug Amina 1, Sadrati Nouari 1, Bouguerra Asma 1, \\ Gaamoune Soufiane ${ }^{1,3}$ and Naili Oumaima ${ }^{1}$ \\ 1: Laboratory of Applied Microbiology, Faculty of Natural Sciences and Life, University Ferhat Abbas Setif1, Setif 19000, Algeria. \\ 2: Department of Natural Sciences and Life, University of Abdel Hafid Boussouf, Mila 43000, Algeria. \\ 3: National Institute of Agriculture Research, Setif 19000, Algeria.
}

\section{ABSTRACT}

Increasingly, the flavonoids becoming the subject of biomedical researches, and many groups have isolated and identified the structures of flavonoids possessing important biological activities. This assess investigates the antimicrobial fight and the antioxidant strengths of flavonoids extracted from Pistacia lentiscus L. leaves. The extract was first analyzed for its phenolic content, then the flavonoids were tested for their antimicrobial capacities by the method of diffusion against fourteen bacteria, two fungi and one yeast. The minimum inhibitory concentration (MIC) and the minimum bactericidal concentration (MBC) of flavonoids were determined using the dilution method on solid mediu m, however, the antioxidant activity was evaluated through the ability of the extract to scavenge DPPH (1,1-diphenyl-2-picrylhydrazyl) radicals and the reducing power essay. The results indicate that flavonoids extract contain other phenolics groups. On the other side, the Antibacterial tests exhibited different activities depending on the strains. Unlike, the MIC and the MBC showed that the flavonoids extract showed a best activity against Candida albicans and Vibrio cholerae. Concerning antioxidant activity, the results indicate a moderate capacity of the flavonoid extract. These preliminary results may justify the use of this plant in the treatment of some diseases and may be exploited for therapeutic purposes.
\end{abstract}

Keywords: Antimicrobial, Antioxidant, Pistacia lentiscus, flavonoids, MIC, MBC.

Article Info: Received 26 Nov 2019; Review Completed 18 Jan 2020; Accepted 27 Jan 2020; Available online 15 Feb 2020

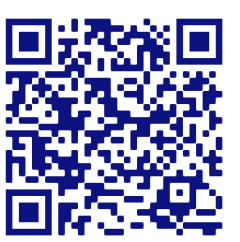

Cite this article as:

Bakli S, Harzallah D, Zerroug A, Sadrati N, Bouguerra A, Gaamoune S, Naili O, Antimicrobial and Antioxidant Activities of Flavonoids Extracted from Pistacia lentiscus L., Leaves, Journal of Drug Delivery and Therapeutics. 2020; 10(1-s):82-89 http://dx.doi.org/10.22270/jddt.v10i1-s.3895

*Address for Correspondence:

Bakli Sabrina, Laboratory of Applied Microbiology, Faculty of Nature and Life Sciences, University Ferhat Abbas Setif1, Setif 19000, Algeria.

\section{INTRODUCTION}

P. lentiscus leaves contain differing types of secondary metabolites. Among these, the most abundant compounds in $P$. lentiscus leaves are reportedly flavonoids that have displayed a powerful antioxidant capacity as well as hepatoprotective, anti-inflammatory, anticancer effects, antimicrobial, antiviral, anticholinesterase, antiinflammatory, antinociceptive, antidiabetic, antitumor, antihyperlipidemic, antiatherosclerotic and hepatoprotective activities and also their beneficial effects in gastrointestinal disorders [1]. Thus, the characterization of bioactive compounds from $P$. lentiscus leaves extracts has relevance for developing nutraceuticals and dietary supplements [2].

Nowadays, the development of resistance of a pathogen to a large number of commonly used antibiotics encourages the search for new antimicrobial agents to fight infections and overcome the resistance and side effects of antimicrobial agent currently available [3]. Due to the alarming increase in the incidence of new and re-emerging infectious diseases, there is a continuing and urgent need to find new antimicrobial compounds with different chemical structures and new mechanisms of action. Another major concern is the development of antibiotic resistance in current clinical use [4]. Flavonoids are increasingly the subject of anti-infectious research. Several flavonoids have antibacterial and antifungal activities [5].

The aim of this study is to evaluate the antioxidant and antimicrobial activities of flavonoids extract obtained from leaves of $P$. lentiscus, in order to find new molecules able to reduce the use of synthetics medicines. 


\section{MATERIAL AND METHODS}

\section{Plant material}

The leaves of P. lentiscus were harvested in July 2014, in Bni Ourtilan - Setif- Algeria. The P. lentiscus leaves were airdried in the shade at room temperature. After drying, the plant material was ground to a fine powder using an electric mill.

\section{Flavonoids extraction}

The powder was taken up three times with $70 \%$ ethanol (raw material: solvent ratio was 1: 10) for 90 minutes at 100 ${ }^{\circ} \mathrm{C}$. The extracts were combined and concentrated under vacuum to collect the aqueous residue $(10 \mathrm{~mL})$, which was extracted with hexane and chloroform, then acidified with 20 $\% \mathrm{H}_{2} \mathrm{SO}_{4}(\mathrm{pH}=5)$ and finally extracted with ethyl acetate. The appearance of an interphase precipitate was observed during extraction with ethyl acetate. The ethyl acetate fraction was taken as a flavonoids fraction for our experiment [6].

\section{Determination of total flavonoids contents}

The flavonoids contents in the extract was estimated by the Aluminium chloride solution according to the method described by Mimica-Dukié (1992) in Maksimović et al. [7]. Briefly, $2 \mathrm{~mL}$ of the methanol solution of the extract was added to $1 \mathrm{~mL}$ of $\mathrm{AlCl}_{3}$ (133 mg crystalline aluminium chloride and $400 \mathrm{mg}$ crystalline sodium acetate were dissolved in $100 \mathrm{~mL}$ of distilled water) in methanol. After 10 minutes, the absorbance was determined at $430 \mathrm{~nm}$. Results were expressed as mg equivalent rutin per gram of extract (mg ER/GE).

\section{Antioxidant activities}

\section{DPPH radical scavenging activity}

The DPPH (1,1-diphenyl-2-picrylhydrazyl) radical scavenging activity of the flavonoids extract was measured according to the procedure described by Sreenivasan $\boldsymbol{e t}$ al. [8]. Briefly; $50 \mu \mathrm{L}$ of the extract at different concentrations were added to $5 \mathrm{~mL}(0,04 \%)$ of DPPH methanolic solution. The decrease in absorbance was determined at $517 \mathrm{~nm}$, after incubation for 30 minutes at laboratory temperature in the dark.

The antiradical activity was expressed as IC $_{50}$ (micrograms per millilitre) and the antiradical dose required to cause a 50 $\%$ of inhibition. A lower $\mathrm{IC}_{50}$ value corresponds to a better antioxidant activity. The ability to scavenge DPPH radical was calculated by employing the subsequent equation:

$$
\text { DPPH scavenging effect }(\%)=\left[\left(A_{0}-A_{1}\right) / A_{0}\right] \times 100
$$

Where $A_{0}$ is the absorbance of the control at 30 minutes and $\mathrm{A}_{1}$ is the absorbance of the sample at 30 minutes. BHT was used as a standard and samples were analyzed in triplicate.

\section{Ferric reducing power (FRP)}

The reducing power of the extract was measured with a method described by Kaur et al. [9]. One millilitre of the extract at different concentrations was mixed with $2.5 \mathrm{~mL}$ of phosphate buffer solution $(0.2 \mathrm{M}, \mathrm{pH} 6.6)$ and $2.5 \mathrm{~mL}$ of potassium ferricyanide $(1 \%)$. The mixture was incubated for 20 minutes at $50{ }^{\circ} \mathrm{C}$. After cooling, $2.5 \mathrm{~mL}$ of $10 \%$ trichloroacetic acid was added and the mixture was centrifuged at $3000 \mathrm{rpm}$ for 10 minutes. $2.5 \mathrm{~mL}$ of supernatant was mixed with $2.5 \mathrm{~mL}$ of distilled water and 0.5 $\mathrm{mL}$ of ferric chloride $(0.1 \%)$. The absorbance of the mixture thus obtained is measured at $700 \mathrm{~nm}$ against a blank.
Higher absorbance indicates higher reducing power. $\mathrm{EC}_{50}$ value $(\mu \mathrm{g}$ extract $/ \mathrm{mL})$ is the effective concentration at which the absorbance was 0.5 for reducing power, obtained by interpolation from linear regression analysis. BHT was used as standards.

\section{Antimicrobial activity test}

\section{Microorganisms}

Evaluation of the antimicrobial activity of extract was performed against fourteen strains of pathogenic bacteria, obtained from the American Type Culture Collection, namely: Staphylococcus aureus ATCC 25923, methicillin resistant Staphylococcus aureus (MRSA) ATCC 43300, Bacillus cereus ATCC 10876, Enterococcus faecalis ATCC 49452, Citrobacter freundii ATCC 8090, Escherichia coli ATCC 25922, Klebsiella pneumoniae ATCC 700603, Salmonella typhi ATCC 14028, Salmonella enterica ATCC43972, Salmonella typhymirium ATCC 13311, Pseudomonas aeruginosa ATCC 27853, Proteus mirabilis ATCC 35659, Vibrio cholerae ATCC 14035 and Listeria innocua CLIP 74915 from Collection Listeria Pasteur Institute.

Two fungi: Aspergillus niger 2CA936, Aspergillus flavus NRRL 3357 and one yeast: Candida albicans ATCC1024. Sabouraud Dextrose agar and Mueller-Hinton agar were used for fungi and bacteria, respectively.

\section{Screening for antibacterial activity}

The screening of antibacterial activity of flavonoids extract of $P$. lentiscus was carried out with agar disc diffusion method [10].

The extract was dissolved in dimethylsulfoxide (DMSO) to a final concentration of $200 \mathrm{mg} / \mathrm{mL}$ and sterilized by filtration through a $0.22 \mu \mathrm{m}$ membrane filter. The bacteria inoculum was prepared by suspending colonies during 24 hours culture. The cell density of each inoculum was adjusted at $10^{8} \mathrm{CFU} / \mathrm{mL}$ suspension with a spectrophotometer (DO=0.08-0.1 $/ \lambda=600 \mathrm{~nm}$ ) [11]. The discs Watt-man paper discs $\mathrm{N}^{\circ} 3$ of $6 \mathrm{~mm}$ diameter were each impregnated with 10 $\mu \mathrm{L}$ and $20 \mu \mathrm{L}$ of extract equal to $2 \mathrm{mg}$ and $4 \mathrm{mg}$ by disc, respectively. The plates were held for 3 hours at $4{ }^{\circ} \mathrm{C}$ for diffusion of extract into the agar [12] [13] and then incubated at $37{ }^{\circ} \mathrm{C}$ for 24 hours. Amoxicillin, Ciprofloxacin, Teicoplanin, Doxycyclin, Pefloxacin, Optochin were used as standards and dimethylsulfoxide as a control.

The activity is determined by the measurement of the inhibitory zone diameter in $\mathrm{mm}$. The antibacterial activity is considered starting from a diameter of $6 \mathrm{~mm}$ or higher, and is classified as follows [14]:

$$
\begin{array}{ll}
\checkmark & \text { Very sensitive: diameter } \geq 20 \mathrm{~mm} ; \\
\checkmark & \text { Sensitive enough: diameter between } 15-19 \mathrm{~mm} ; \\
\checkmark & \text { Sensitive: diameter between 09-14 mm; } \\
\checkmark & \text { Resistant: diameter } \leq 08 \mathrm{~mm} .
\end{array}
$$

\section{Screening for Antifungal activity}

The antifungal activity was tested by disc diffusion method [15]. The Sabouraud plates were inoculated with each fungal culture.

The spore suspension was prepared in saline solution $(0.9 \%$ $\mathrm{NaCl}$ ), adjusted to a concentration of $10^{6}$ spores/mL [15] [16] corresponding to 0.15 to 0.17 absorption at $530 \mathrm{~nm}$ [15]. One hundred microliter of suspension was placed over agar in Petri dishes and dispersed using a sterile swab. Then, the sterile paper discs ( $6 \mathrm{~mm}$ diameter) were placed on agar to load $10 \mu \mathrm{L}$ and $20 \mu \mathrm{L}$ equal to $2 \mathrm{mg}$ and $4 \mathrm{mg}$ by disc, respectively. And the plates were held for $3 \mathrm{~h}$ at $4{ }^{\circ} \mathrm{C}$ for diffusion of extracts into the agar and then incubated at $37^{\circ} \mathrm{C}$ 
for 48 hours for C. albicans and 72 hours at $28{ }^{\circ} \mathrm{C}$ for the fungi.

Nystatin, clotrimazon and amphotericin were used as standards and dimethylsulfoxide as a control.

\section{Determination of Bactericidal and Fungicidal Activity}

\section{Minimum inhibition concentration (MIC)}

The MIC of the extract was determined using agar dilution method that has been already described by [17]. One milliliter of different concentrations of extract was added to $14 \mathrm{~mL}$ of nutrient agar to make the final concentration ranging from $10 \mathrm{mg} / \mathrm{mL}$ to $0.05 \mathrm{mg} / \mathrm{mL}$. Standardized inocula approximately containing $1 \times 10^{4} \mathrm{CFU} / \mathrm{mL}$ [18] were spotted on solidified plates containing various concentrations of the extract. The lowest concentration of extract inhibiting any visible bacterial or fungal growth after an incubation period of $24 \mathrm{~h}$ at $37{ }^{\circ} \mathrm{C}$ and $48 \mathrm{~h}$ at $28^{\circ} \mathrm{C}$, respectively, was taken as the minimum inhibitory concentration [17] [19].

Minimum Bactericidal Concentration (MBC) and Minimal Fungicidal Concentration (MFC)

Samples were taken from the nutrient agar plates that showed no visible growth after 24 hours incubation and sub cultured into tubes containing nutrient broth. The least concentration that did not produce growth after 24 hours was regarded as the minimal bactericidal concentration [17].

\section{Statistical analysis}

Experiments were carried out in triplicate and expressed as the mean \pm standard deviation, data were analysed and compared using the one-way ANOVA and Tukey Multiple Comparison with $95 \%$ confidence limits $(\mathrm{P}<0.05)$, using Graphpad prism 5 Demo Software.

\section{RESULTS AND DISCUSSION}

Flavonoids extracted from the leaves of $P$. lentiscus reach the yield of $3.91 \%$ with $278.507 \pm 0.377 \mathrm{mg}$ ER/GE of total flavonoids.

\section{Antioxidant activity}

The isolated compounds were screened for antioxidant activity. The results of DPPH test were showed in figure 1.

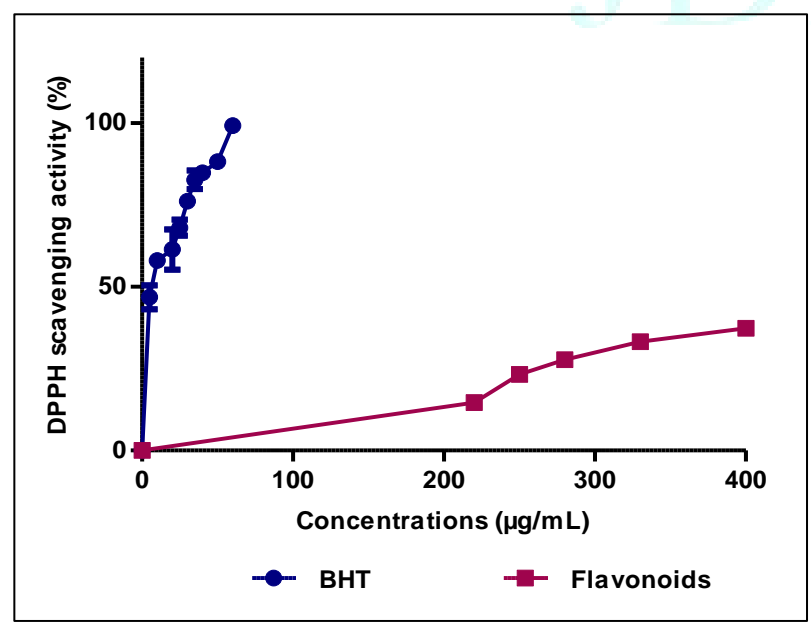

Figure 1: Scavenging activity on the DPPH radical of flavonoids extracted from leaves of $P$. Lentiscus

The $\mathrm{IC}_{50}$ of flavonoids extract was $455 \pm 0,048 \mu \mathrm{g} / \mathrm{mL}^{* * *}$ against $4,31 \pm 0,79 \mu \mathrm{g} / \mathrm{mL}$ for BHT.

Studies have recently shown that flavonoids have an antioxidant effect on human health. These compounds scavenge free radicals and chelate metal thus avoiding the Fenton reaction to occur [20].The role of antioxidants in the inhibition of oxidant processes occurring in living organisms consists of scavenging free radicals and quenching singlet oxygen, disconnection of radical reactions, chelate metals which catalyze the oxidation process, inhibition of certain enzymes (eg., oxidases). Flavonoids are active in these processes [21]. However the weakness of our extract due to the presence of other molecules which affect the ability of scavenging in terms of hydrogen donating ability.

In addition, it is known that only flavonoids having a certain structure and in particular a hydroxyl position in the molecule can act as proton donors and show a radical scavenging activity [22] [23].

The reducing capacity of a compound can be considered as an important indicator of its antioxidant activity [24]. Their absorption values increased with increase in concentration, showing that as the concentration of the extract was increased their ability to reduce $\mathrm{Fe}^{3+}$ to $\mathrm{Fe}^{2+}$ was also increased. Figure 2 shows the dose-dependent curve for the reducing power of the extract:

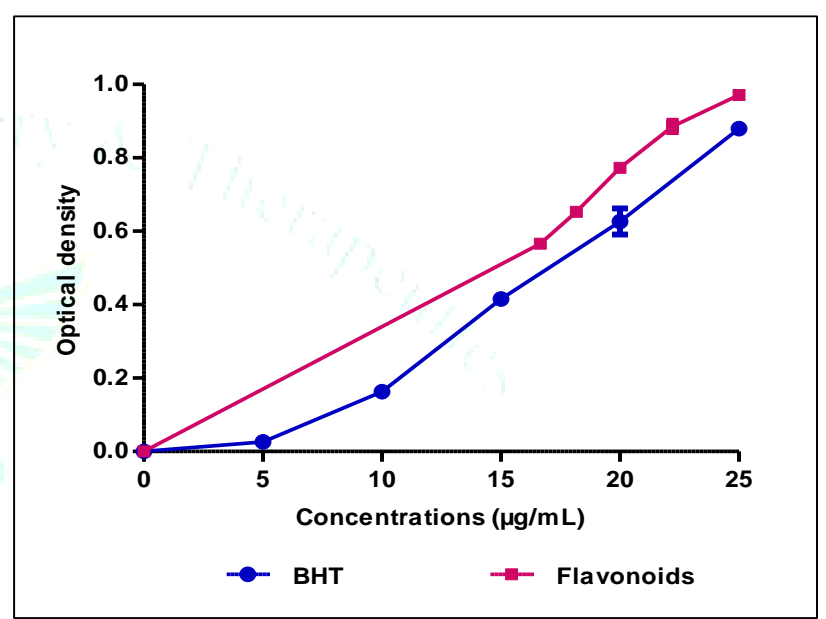

Figure 2: Reducing power of flavonoids extracted from the leaves of $P$. lentiscus

An increase in absorbance corresponds to an increase in the reducing power of the extract tested. Flavonoids showed increased ferric reducing power with the increasing concentration with $\mathrm{EC}_{50}$ correspond to $15.0 \pm 0.001 \mu \mathrm{g} / \mathrm{mL}$ against $16.0 \pm 0.001 \mu \mathrm{g} / \mathrm{mL}$ of BHT. The extract expressed electron donating activity and its power was equal to BHT.

High reducing power of flavonoids extract suggested its remarkable potency to donate electrons to reactive free radicals, thus, converting them into more stable non-reactive species and finally terminate the free radical chain reaction [25]. It was confirmed that the hydroxyl groups at C-3' and C4' of the B-ring to be more active in reducing iron concentration [26]; this may explain our results.

The study of correlation between radical scavenging power and the capacity of metal ions chelation seems interesting. Firstly because oxidative stress is the cause of formation of free radicals through metals transition (iron, copper) via Fenton reaction [20]. Secondly, the evaluation of the plant antioxidant effect is made by combining the results of at least two in vitro assays [27].

\section{Antimicrobial activity}

The results obtained from antimicrobial assay are presented in Table 1, 2 and 3: 
Table 1: Antibacterial activities of flavonoids, pure phenolic compounds, standards and control

\begin{tabular}{|c|c|c|c|c|c|c|c|c|c|c|c|c|c|c|}
\hline \multirow{2}{*}{ 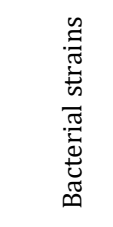 } & \multicolumn{14}{|c|}{ Zone of inhibition (mm) } \\
\hline & $\dot{\infty}$ & 峦 &  & $\infty \frac{y}{\tilde{s}}$ & แิ่ ธี & 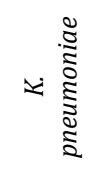 & $v \frac{\pi}{\frac{\pi}{2}}$ & vi $\frac{\overline{0}}{\frac{0}{2}}$ & 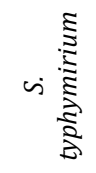 & ن & a: $\frac{8}{\frac{8}{5}}$ & Q: & $>\frac{\frac{d}{\frac{0}{2}}}{\frac{8}{2}}$ & 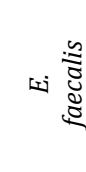 \\
\hline \multicolumn{15}{|c|}{ Flavonoids ( $200 \mathrm{mg} / \mathrm{mL}$ ) } \\
\hline $10 \mu \mathrm{L}$ & $\begin{array}{c}12,7 \pm \\
0,47^{a}\end{array}$ & $\begin{array}{c}9,0 \pm 0 \\
0^{\mathrm{a}}\end{array}$ & $-/-$ & $\begin{array}{r}8,0 \pm \\
0,00^{\mathrm{a}}\end{array}$ & $-/-$ & $-/-$ & $\begin{array}{c}9,0 \pm 0 \\
0^{\mathrm{a}}\end{array}$ & $-/-$ & $-/-$ & $-/-$ & $\begin{array}{c}9,0 \pm 0 \\
0^{\mathrm{a}}\end{array}$ & $\begin{array}{c}7,3 \pm 0 \\
47^{\mathrm{a}}\end{array}$ & $\begin{array}{l}10,3 \pm \\
0,47^{a}\end{array}$ & $-/-$ \\
\hline $20 \mu \mathrm{L}$ & $\begin{array}{l}18,8 \pm \\
0,24 c\end{array}$ & $\begin{array}{l}13,0 \pm \\
0,41^{b}\end{array}$ & $\begin{array}{c}8,3 \pm 0 \\
, 47 a\end{array}$ & $\begin{array}{r}14,7 \\
\pm 0,47 \mathrm{c}\end{array}$ & $\begin{array}{c}8,8 \pm 0 \\
, 24 \mathrm{a}\end{array}$ & $-/-$ & $\begin{array}{l}11,5 \pm \\
0,41^{b}\end{array}$ & $\begin{array}{c}8,7 \pm 0 \\
47 a\end{array}$ & $-/-$ & $-/-$ & $\begin{array}{l}14,0 \pm \\
0,00 \mathrm{e}\end{array}$ & $\begin{array}{c}13,0 \pm 0 \\
, 0^{\mathrm{b}}\end{array}$ & $\begin{array}{l}12,7 \pm \\
0,47 \mathrm{~b}\end{array}$ & $\begin{array}{c}8,3 \pm 0 \\
47 a\end{array}$ \\
\hline \multicolumn{15}{|c|}{$\begin{array}{l}\text { Pure phenolic } \\
\text { compounds }\end{array}$} \\
\hline \multicolumn{15}{|l|}{$\begin{array}{l}\text { Tannic } \\
\text { acid }\end{array}$} \\
\hline $10 \mu \mathrm{L}$ & $\begin{array}{c}13,8 \pm \\
0,3^{\mathrm{a}}\end{array}$ & $\begin{array}{c}14,0 \pm \\
0,0^{\mathrm{b}}\end{array}$ & $-/-$ & $\begin{array}{c}10,0 \pm \\
0,6^{b}\end{array}$ & $-/-$ & $-/-$ & $\begin{array}{c}18,0 \pm \\
0,0^{c}\end{array}$ & $\begin{array}{c}12,0 \pm \\
0,0^{\mathrm{b}}\end{array}$ & $-/-$ & $-/-$ & $\begin{array}{c}12,0 \pm \\
0,0^{c}\end{array}$ & $\begin{array}{c}14,0 \pm 0 \\
, 0^{\mathrm{b}}\end{array}$ & $\begin{array}{c}15,5 \pm \\
0,7 \mathrm{c}\end{array}$ & $\begin{array}{c}8,0 \pm 0 \\
0^{\mathrm{a}}\end{array}$ \\
\hline $20 \mu \mathrm{L}$ & $\begin{array}{c}17,3 \pm \\
0,6^{b}\end{array}$ & $\begin{array}{c}18,0 \pm \\
0,0^{c}\end{array}$ & $\begin{array}{c}11,3 \pm \\
0,6^{b}\end{array}$ & $\begin{array}{c}16,5 \pm \\
0,5^{d}\end{array}$ & $\begin{array}{c}12,0 \pm \\
0,0^{\mathrm{b}}\end{array}$ & $-/-$ & $\begin{array}{c}20,3 \pm \\
0,6^{d}\end{array}$ & $\begin{array}{c}15,7 \pm \\
0,6^{c}\end{array}$ & $-/-$ & $-/-$ & $\begin{array}{c}13,0 \pm \\
0,0^{d}\end{array}$ & $\begin{array}{c}18,2 \pm \\
0,8^{c}\end{array}$ & $\begin{array}{c}22,0 \pm \\
0,0^{d}\end{array}$ & $\begin{array}{c}12,3 \pm \\
0,6^{b}\end{array}$ \\
\hline \multicolumn{15}{|l|}{$\begin{array}{l}\text { Galic } \\
\text { acid }\end{array}$} \\
\hline $10 \mu \mathrm{L}$ & $-/-$ & $-/-$ & $-/-$ & $-/-$ & $-/-$ & $-/-$ & $-/-$ & $-/-$ & $-/-$ & $-/-$ & $-/-$ & $-/-$ & $-/-$ & $-/-$ \\
\hline $20 \mu \mathrm{L}$ & $-/-$ & $-/-$ & $-/-$ & $-/-$ & $-/-$ & $-/-$ & $-/-$ & $-/-$ & $-/-$ & $-/-$ & $-/-$ & $\begin{array}{c}8,0 \pm 0 \\
0^{\mathrm{a}}\end{array}$ & $\begin{array}{c}11,0 \pm \\
0,0^{\mathrm{a}}\end{array}$ & $-/-$ \\
\hline \multicolumn{15}{|l|}{$\begin{array}{l}\text { Quercet } \\
\text { in }\end{array}$} \\
\hline $10 \mu \mathrm{L}$ & $-/-$ & $-/-$ & $-/-$ & $-/-$ & $-/-$ & $-/-$ & $-/-$ & $-/-$ & $-/-$ & $-/-$ & $-/-$ & $-/-$ & $-/-$ & $-/-$ \\
\hline \multicolumn{15}{|l|}{$\begin{array}{l}\text { Standar } \\
\text { ds }\end{array}$} \\
\hline $\begin{array}{l}\text { Amoxici } \\
\text { llin }\end{array}$ & $\begin{array}{c}30,0 \pm \\
0,0^{\mathrm{d}}\end{array}$ & $\begin{array}{c}9,0 \pm 0 \\
0^{\mathrm{a}}\end{array}$ & $\begin{array}{c}23,0 \pm \\
0,0^{\mathrm{d}}\end{array}$ & $-/-$ & $\begin{array}{c}12,0 \pm \\
0,0^{\mathrm{b}}\end{array}$ & $-/-$ & $\begin{array}{c}22,0 \pm \\
0,0^{\mathrm{e}}\end{array}$ & $\begin{array}{c}23,0 \pm \\
0,0^{\mathrm{f}}\end{array}$ & $-/-$ & $-/-$ & $-/-$ & $\begin{array}{c}39,67 \pm \\
0,47^{f}\end{array}$ & $\begin{array}{c}22,0 \pm \\
0,0^{\mathrm{d}}\end{array}$ & $\begin{array}{c}22,0 \pm \\
0,0^{\mathrm{d}}\end{array}$ \\
\hline $\begin{array}{l}\text { Ciproflo } \\
\text { xacin }\end{array}$ & $\begin{array}{l}34,7 \pm \\
0,47 \text { ef }\end{array}$ & $\begin{array}{c}35,0 \pm \\
0,0^{\mathrm{e}}\end{array}$ & $\begin{array}{c}32,0 \pm \\
0,0^{\mathrm{e}}\end{array}$ & $\begin{array}{r}37,3 \pm \\
0,47 \mathrm{~g}\end{array}$ & $\begin{array}{c}46,0 \pm \\
0,0 \mathrm{e}\end{array}$ & $\begin{array}{c}32,0 \pm \\
0,0\end{array}$ & $\begin{array}{c}32,0 \pm \\
0,0 \mathrm{~g}\end{array}$ & $\begin{array}{r}26,7 \pm \\
0,47 \mathrm{~g}\end{array}$ & $\begin{array}{c}45,3 \pm \\
0,47\end{array}$ & $\begin{array}{c}51,3 \pm \\
0,47\end{array}$ & $\begin{array}{c}37,7 \pm \\
0,47 \mathrm{~g}\end{array}$ & $\begin{array}{c}33,67 \pm \\
0,47 \mathrm{e}\end{array}$ & $\begin{array}{l}49,0 \pm \\
0,82^{g}\end{array}$ & $\begin{array}{r}31,7 \pm \\
0,47^{f}\end{array}$ \\
\hline $\begin{array}{l}\text { Teicopl } \\
\text { anin }\end{array}$ & $\begin{array}{l}19,7 \pm \\
0,47 \mathrm{c}\end{array}$ & $\begin{array}{c}20,0 \pm \\
0,0^{\mathrm{d}}\end{array}$ & $\begin{array}{c}21,0 \pm \\
0,0^{c}\end{array}$ & $\begin{array}{c}15,0 \pm \\
0,0^{c}\end{array}$ & $-/-$ & $-/-$ & $\begin{array}{r}22,3 \pm \\
0,47 \mathrm{e}\end{array}$ & $\begin{array}{c}20,0 \pm \\
0,0^{\mathrm{d}}\end{array}$ & $-/-$ & $\begin{array}{c}16,8 \pm \\
0,62\end{array}$ & $-/-$ & $\begin{array}{c}25,0 \pm 0 \\
, 0^{\mathrm{d}}\end{array}$ & $\begin{array}{c}26,0 \pm \\
0,0 \mathrm{e}\end{array}$ & $\begin{array}{c}20,0 \pm \\
0,0^{c}\end{array}$ \\
\hline $\begin{array}{l}\text { Doxycy } \\
\text { clin }\end{array}$ & $\begin{array}{c}36,0 \pm \\
0,0^{f}\end{array}$ & $\begin{array}{c}39,0 \pm \\
1,0^{f}\end{array}$ & $\begin{array}{c}34,0 \pm \\
0,0^{f}\end{array}$ & $\begin{array}{c}26,0 \pm \\
0,0 \mathrm{e}\end{array}$ & $\begin{array}{c}30,0 \pm \\
0,0 \mathrm{c}\end{array}$ & $\begin{array}{c}10,0 \pm \\
0,0\end{array}$ & $\begin{array}{c}50,0 \pm \\
0,0^{\mathrm{h}}\end{array}$ & $\begin{array}{c}30,0 \pm \\
0,0^{\mathrm{h}}\end{array}$ & $\begin{array}{c}25,0 \pm \\
0,0\end{array}$ & $\begin{array}{c}28,0 \pm \\
0,0\end{array}$ & $\begin{array}{c}10,0 \pm \\
0,0^{\mathrm{b}}\end{array}$ & $\begin{array}{c}44,0 \pm 0 \\
, 0 \mathrm{~g}\end{array}$ & $\begin{array}{c}44,0 \pm \\
0,0^{f}\end{array}$ & $\begin{array}{c}37,0 \pm \\
0,0 \mathrm{~g}\end{array}$ \\
\hline $\begin{array}{l}\text { Pefloxa } \\
\text { cin }\end{array}$ & $\begin{array}{c}33,7 \pm \\
0,6^{\mathrm{e}}\end{array}$ & $\begin{array}{c}35,5 \pm \\
0,5^{e}\end{array}$ & $-/-$ & $\begin{array}{c}30,5 \pm \\
0,7^{f}\end{array}$ & $\begin{array}{c}44,0 \pm \\
0,0^{\mathrm{d}}\end{array}$ & $\begin{array}{c}26,0 \pm \\
0,0\end{array}$ & $\begin{array}{c}25,0 \pm \\
0,0^{f}\end{array}$ & $\begin{array}{c}22,0 \pm \\
0,0^{\mathrm{e}}\end{array}$ & $\begin{array}{c}40,0 \pm \\
0,0\end{array}$ & $\begin{array}{c}46,0 \pm \\
0,0\end{array}$ & $\begin{array}{c}22,0 \pm \\
0,0^{f}\end{array}$ & $\begin{array}{c}40,0 \pm 0 \\
, 0^{f}\end{array}$ & $\begin{array}{c}44,0 \pm \\
0,0^{\mathrm{f}}\end{array}$ & $\begin{array}{c}30,0 \pm \\
0,0^{\mathrm{e}}\end{array}$ \\
\hline $\begin{array}{l}\text { Optochi } \\
\text { n }\end{array}$ & $-/-$ & $-/-$ & $-/-$ & $-/-$ & $-/-$ & $-/-$ & $-/-$ & $-/-$ & $-/-$ & $-/-$ & $-/-$ & $-/-$ & $-/-$ & $-/-$ \\
\hline Control & $\begin{array}{l}\text { No } \\
\text { inhibit } \\
\text { ion }\end{array}$ & $\begin{array}{l}\text { No } \\
\text { inhibit } \\
\text { ion }\end{array}$ & $\begin{array}{l}\text { No } \\
\text { inhibi } \\
\text { tion }\end{array}$ & $\begin{array}{l}\text { No } \\
\text { inhibi } \\
\text { tion }\end{array}$ & $\begin{array}{l}\text { No } \\
\text { inhibi } \\
\text { tion }\end{array}$ & $\begin{array}{l}\text { No } \\
\text { inhibi } \\
\text { tion }\end{array}$ & $\begin{array}{l}\text { No } \\
\text { inhibit } \\
\text { ion }\end{array}$ & $\begin{array}{l}\text { No } \\
\text { inhibi } \\
\text { tion }\end{array}$ & $\begin{array}{l}\text { No } \\
\text { inhibi } \\
\text { tion }\end{array}$ & $\begin{array}{l}\text { No } \\
\text { inhibi } \\
\text { tion }\end{array}$ & $\begin{array}{l}\text { No } \\
\text { inhibi } \\
\text { tion }\end{array}$ & $\begin{array}{l}\text { No } \\
\text { inhibiti } \\
\text { on }\end{array}$ & $\begin{array}{l}\text { No } \\
\text { inhibit } \\
\text { ion }\end{array}$ & $\begin{array}{l}\text { No } \\
\text { inhibi } \\
\text { tion }\end{array}$ \\
\hline
\end{tabular}

-/-: No zone of inhibition

In the same column, means followed by the same letters are not significantly different $(\mathrm{p}<0.05)$ 
Table 2: Antifungal activities of flavonoids, pure phenolic compounds, standards and control

\begin{tabular}{|c|c|c|c|}
\hline \multirow[b]{2}{*}{$\begin{array}{l}\text { Fungal } \\
\text { strains }\end{array}$} & \multicolumn{3}{|c|}{ Zone of inhibition (mm) } \\
\hline & Aspergillus flavus & Aspergillus niger & Candida albicans \\
\hline \multicolumn{4}{|c|}{ Flavonoids $(200 \mathrm{mg} / \mathrm{mL})$} \\
\hline $10 \mu \mathrm{L}$ & $-/-$ & $-/-$ & $12,67 \pm 0,47 \mathrm{~b}$ \\
\hline $20 \mu \mathrm{L}$ & $-/-$ & $-/-$ & $14,0 \pm 0,0^{\mathrm{b}}$ \\
\hline \multicolumn{4}{|c|}{$\begin{array}{l}\text { Purphenolic compounds } \\
\text { Tannic acid }\end{array}$} \\
\hline $10 \mu \mathrm{L}$ & $11,3 \pm 0,6^{a}$ & $-/-$ & $18,8 \pm 1,0^{d}$ \\
\hline $20 \mu \mathrm{L}$ & $12,9 \pm 0,3^{b}$ & $-/-$ & $25,0 \pm 0,0^{\mathrm{e}}$ \\
\hline \multicolumn{4}{|l|}{ Galic acid } \\
\hline $10 \mu \mathrm{L}$ & $-/-$ & $-/-$ & $-/-$ \\
\hline $20 \mu \mathrm{L}$ & $-/-$ & $-/-$ & $-/-$ \\
\hline \multicolumn{4}{|l|}{ Quercetin } \\
\hline $10 \mu \mathrm{L}$ & $-/-$ & $-/-$ & $-/-$ \\
\hline $20 \mu \mathrm{L}$ & $-/-$ & $-/-$ & $-/-$ \\
\hline \multicolumn{4}{|l|}{ Standards } \\
\hline Amphotericin & $16,67 \pm 0,29^{d}$ & $17,17 \pm 0,29 \mathrm{c}$ & $15,67 \pm 0,29 c$ \\
\hline Clotrimazon & $23,50 \pm, 50^{e}$ & $15,67 \pm 0,29 b$ & $44,67 \pm 0,58^{\mathrm{f}}$ \\
\hline Nystatin & $15,17 \pm 0,29 \mathrm{c}$ & $9,17 \pm 0,29$ a & $9,17 \pm 0,29$ a \\
\hline Control & No inhibition & No inhibition & No inhibition \\
\hline
\end{tabular}

-/-: No zone of inhibition;

In the same column, means followed by the same letters are not significantly different $(\mathrm{p}<0.05)$

Table 3: MIC and MBC of flavonoids and pure phenolic compounds against the tested microbial strains

\begin{tabular}{|c|c|c|c|c|c|c|c|c|c|c|c|c|c|c|c|c|}
\hline \multirow[b]{3}{*}{ Strains } & \multicolumn{16}{|c|}{ MIC et MBC $/ \mathrm{MFC}(\mathrm{mg} / \mathrm{mL})$} \\
\hline & \multicolumn{14}{|c|}{ Bacterial strains } & \multicolumn{2}{|c|}{ Fungal strains } \\
\hline & vi $\frac{\mathfrak{d}}{\grave{\Xi}}$ & 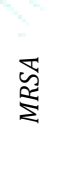 & $\infty \frac{2}{0}$ & 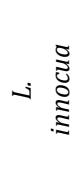 & 떠 & $\downarrow i \frac{\tilde{\Xi}}{\tilde{\Xi}}$ & si $\frac{\pi}{2}$ & 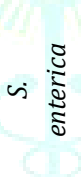 & 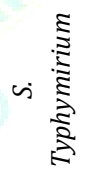 & ن & 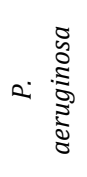 & 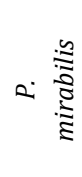 & $>\frac{\frac{8}{2}}{\frac{2}{0}}$ & 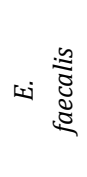 & $ن . \underset{\tilde{\Xi}}{\tilde{\Xi}}$ & 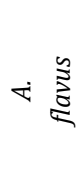 \\
\hline \multicolumn{17}{|l|}{ Flavonoids } \\
\hline MIC & 2 & 0,5 & 1 & 5 & 4 & NT & 0,6 & 5 & NT & NT & 3 & 3 & 0,3 & 5 & 0,1 & NT \\
\hline $\mathrm{MBC} / \mathrm{MFC}$ & 3 & 1 & 6 & 5 & 5 & NT & 3 & 6 & NT & NT & 5 & 3 & 0,5 & 5 & 5 & NT \\
\hline \multicolumn{17}{|c|}{$\begin{array}{l}\text { Pure phenolic compounds } \\
\text { Tannic acid }\end{array}$} \\
\hline MIC & 0,4 & 0,3 & 0,3 & 1 & $>10$ & NT & $\begin{array}{c}<50 \\
\text { ug }\end{array}$ & 1 & NT & NT & 0,9 & 0,1 & 0,1 & 1 & 0,1 & $>10$ \\
\hline $\mathrm{MBC} / \mathrm{MFC}$ & 0,4 & 0,3 & 0,6 & 2 & $>10$ & NT & 3 & 8 & NT & NT & 0,9 & 0,3 & 0,1 & 5 & 5 & $>10$ \\
\hline \multicolumn{17}{|l|}{ Galic acid } \\
\hline MIC & NT & NT & NT & NT & NT & NT & NT & NT & NT & NT & NT & 10 & 8 & NT & NT & NT \\
\hline $\mathrm{MBC} / \mathrm{MFC}$ & NT & NT & NT & NT & NT & NT & NT & NT & NT & NT & NT & 10 & 8 & NT & NT & NT \\
\hline \multicolumn{17}{|l|}{ Quercetin } \\
\hline MIC & NT & NT & NT & NT & NT & NT & NT & NT & NT & NT & NT & NT & NT & NT & NT & NT \\
\hline $\mathrm{MBC} / \mathrm{MFC}$ & NT & NT & NT & NT & NT & NT & NT & NT & NT & NT & NT & NT & NT & NT & NT & NT \\
\hline \multicolumn{17}{|c|}{ Antibiotic standards } \\
\hline Amoxicillin & NT & NT & NT & NT & NT & NT & NT & NT & NT & NT & NT & NT & NT & NT & NT & NT \\
\hline $\begin{array}{l}\text { Ciprofloxa } \\
\text { cin }\end{array}$ & NT & NT & NT & NT & NT & NT & NT & NT & NT & NT & NT & NT & NT & NT & NT & NT \\
\hline $\begin{array}{l}\text { Teicoplani } \\
\mathrm{n}\end{array}$ & NT & NT & NT & NT & NT & NT & NT & NT & NT & NT & NT & NT & NT & NT & NT & NT \\
\hline Doxycyclin & NT & NT & NT & NT & NT & NT & NT & NT & NT & NT & NT & NT & NT & NT & NT & NT \\
\hline Pefloxacin & NT & $\mathrm{NT}$ & NT & NT & NT & NT & NT & NT & NT & NT & NT & NT & NT & NT & NT & NT \\
\hline Optochin & NT & $\mathrm{NT}$ & NT & NT & NT & NT & NT & NT & NT & NT & NT & NT & NT & NT & NT & NT \\
\hline \multicolumn{17}{|c|}{ Antifongic standards } \\
\hline $\begin{array}{l}\text { Amphoteri } \\
\text { cin }\end{array}$ & NT & NT & NT & NT & NT & NT & NT & NT & NT & NT & NT & NT & NT & NT & NT & NT \\
\hline $\begin{array}{l}\text { Clotrimaz } \\
\text { on }\end{array}$ & NT & NT & NT & NT & NT & NT & NT & NT & NT & NT & NT & NT & NT & NT & NT & NT \\
\hline Nystatin & NT & NT & NT & NT & NT & NT & NT & NT & NT & NT & NT & NT & NT & NT & NT & NT \\
\hline
\end{tabular}


The first table (table 1) indicates that the flavonoids extract is weak to moderate inhibitory activities against the tested bacteria even zero against some strains. The flavonoids at 4 $\mathrm{mg} /$ disc showed variable inhibitory activity against all bacteria (Gram-positive and Gram-negative) with inhibition zone diameters ranging from $8.33-18.83 \mathrm{~mm}$. An extract is considered active when it reveals a zone of inhibition greater than or equal to $9 \mathrm{~mm}$ [14]. Furthermore, the assayed extract indicates no activity against tested fungi $A$. niger and A. flavus (Table 2), but showed an important antifungal activity against $C$. albicans with inhibition zone of $14.0 \pm 0.0$ $\mathrm{mm}$. This inhibition was low compared to Amphotericin.

Antimicrobial activity of pure phenolic compounds showed that tannic acid has more impact on $V$. cholerae $(22.0 \pm 0.0$ $\mathrm{mm}$ ) (Table 1). Galic acid has showed minimum zone of inhibition on $P$. mirabilis $(8.0 \pm 0.0 \mathrm{~mm})$ and $V$. Cholera $(11.0 \pm 0.0 \mathrm{~mm})$. On the other hand tannic acid showed significant activity against $C$. albicans $(25.0 \pm 0.0 \mathrm{~mm})$ compared to standards nystatin $(9.17 \pm 0.29 \mathrm{~mm})$ and amphotericin $(15.67 \pm 0.29 \mathrm{~mm})$ and low activity against $A$. flavus $(12.9 \pm 0.3 \mathrm{~mm})$ (Table 2$)$ while quercetin shows no inhibitory effect.

Among the six different types of antibiotics used in the study, Ciprofloxacin has a broad spectrum of activity on all species of human pathogenic bacteria and the widest zone of inhibition was observed against $C$. freundii $(51.33 \pm 0.47 \mathrm{~mm})$ (Table 1). Whereas the minimum zone of inhibition was exhibited in Amoxicillin $(9.0 \pm 0.0 \mathrm{~mm})$ against MRSA. Optochin has no antibacterial activity on the selected test organisms.

The MICs results are not in good agreement with those of the antibiogram for all tested species (Table 3); since the smallest MIC was observed with C. albicans $(0.1 \mathrm{mg} / \mathrm{mL})$ and the latter has small zone of inhibition $(14.0 \pm 0.0 \mathrm{~mm})$, when $S$. aureus showed a high MIC $(2 \mathrm{mg} / \mathrm{mL})$ and the largest zone of inhibition $(18.83 \pm 0.24 \mathrm{~mm})$.

These results show that the flavonoids extract has a lower value of MIC $0.1 \mathrm{mg} / \mathrm{mL}$ on the fungal strain C. albicans, also showed a remarkable activity on $V$. Cholera with value of MBC $0.3 \mathrm{mg} / \mathrm{mL}$ and the best activity were obtained with tannic acid with MIC value of $<50 \mathrm{ug} / \mathrm{mL}$ against $S$. typhi and MBC $0.1 \mathrm{mg} / \mathrm{mL}$ against $V$. Cholera.

In this study, we find out that the extract could give higher inhibition to Gram-positive bacteria compared to Gramnegative bacteria (Table 1). These results can be explain that Gram-negative microorganisms are typically more resistant to antimicrobial agents than Gram-positive bacteria. This has long been explained by the presence of an outer-membrane permeability barrier in Gram-negative bacteria, which limits access of the antimicrobial agents to their targets in the bacterial cells [28] [29]. In addition, the periplasm contains enzymes that destroy foreign molecules introduced from the outside [30].

Antimicrobial activity that is observed in this study is due to the presence of flavonoids compounds in this extract because flavonoids are known for their antimicrobial activity against a wide range of microorganisms [31] [32] and they are multiple cellular targets and may apply to different components and functions in the bacterial cell [33] [34]. Extracts of various medicinal plants containing phenolics and flavonoids have been previously reported to possess antimicrobial activity [35] [36].The presence of these compounds might contribute to antimicrobial activity of $P$. lentiscus leaves since Cushnie et Lamb [37] reported that flavonoids (kaempferol, myricetin, naringin, quercetin and rutin) have antimicrobial activity against human pathogenic microorganisms with some mechanisms of action such as inhibition of nucleic acid synthesis, cytoplasmic membrane function by perforation mechanism and energy metabolism [35] [37] [38]. The antimicrobial activity of the flavonoids extract of $P$. lentiscus leaves might be due to one of the mechanisms of action mentioned above.

The results of the present study are quite encouraging as the flavonoids extract exhibited antimicrobial activity against most of the pathogens. However, the antimicrobial activity varies widely, depending on the microorganism.

\section{CONCLUSION}

Recently there is a huge interest in the use of plant material as an alternative method to control pathogenic microorganism and antioxidant agents.

This study shows that flavonoids extract of $P$. lentiscus leaves was potent as antimicrobial substances and powerful chelating capacity. It is suggested that could be considered as source of antimicrobial and antioxidant agent which might be applied in pharmaceutical and cosmetic products.

Further studies are needed to purify and investigate the molecular composition of this natural product which constitutes an alternative of the synthetic medicines.

\section{REFERENCES}

[1] Bozorgi M, Memariani Z, Mobli M, Salehi Surmaghi MH, ShamsArdekani MR, Rahimi R, Five Pistacia species ( $P$. vera, $P$. atlantica, P. terebinthus, P. khinjuk, and P. lentiscus): A Review of Their Traditional Uses, Phytochemistry, and Pharmacology, The Scientific World Journal, 2013; 1-32.

[2] Rodríguez-Pérez C, Quirantes-Piné R, Amessis-Ouchemoukh N, Madani K, Segura-Carretero A, Fernández-Gutierrez A, A metabolite-profiling approach allows the identification of new compounds from Pistacia lentiscus leaves, Journal of Pharmaceutical and Biomedical Analysis, 2013; 77:167-174.

[3] Ali-Shtayeh MS, YaghmourReem M-R, Faidi YR, Salem k, Al-Nuri MA, Antimicrobial activity of 20 plants used in folkloric medicine in the Palestinian area, Journal of Ethnopharmacology, 1998; 60:265-271.

[4] Rojas R, Bustamante B, Bauer J, Fernández I, Albán J, Lock O, Antimicrobial activity of selected Peruvian medicinal plants, Journal of Ethnopharmacology, 2003; 88:199-204.

[5] Jayshree N, Narayanan N, Sriram L, Antibacterial, antifungal and antimycobacterial studies on some synthetic dimethoxy flavones, Asian Journal of Pharmaceutical and Clinical Research, 2012; 5 (1):101-103.

[6] Chirikova N K, Olennikov DN, Tankhaeva LM, Quantitative Determination of Flavonoid Content in the Aerial Part of Baical Scullcap (Scutellaria baicalensis Georgi), Russian Journal of Bioorganic Chemistry, 2010; 36 (7):915-922.

[7] Maksimović Z, Malencié ND, Kovacevié N, Polyphenol contents and antioxidant activity of Maydis stigma extracts, Bioresource Technology, 2004; 96 (8):873-877.

[8] Sreenivasan S, Ibrahim D, Kassim MJNM, Free radical Scavenging Activity and Total Phenolic Compounds of Gracilaria Changii, International Journal of Natural and Engineering Sciences, 2007; 1 (3):115-117.

[9] Kaur G, Jabbar Z, Athar M, Alam MS, Punica granatum (pomegranate) flower extract possesses potent antioxidant activity and abrogate Fe-NTA induced hepatotoxicity in mice, Food Chemical Toxicology, 2006; 44:984-993.

[10] Benhammou N, AlikBenkkara F, Panovska TK, Antioxidant and antimicrobial activities of the Pistacia lentiscus and Pistacia atlantica extracts, African Journal of Pharmacy and Pharmacology, 2008; 2 (2):022-028. 
[11] Haddouchi F, Lazouni HA, Meziane A, Benmansour A, Etude physicochimique et microbiologique de l'huile essentielle de Thymus fontanesii Boiss \& Reut, Afrique SCIENCE, 2009; 05(2):246-259.

[12] Ali NAA, Julich W-D, Kusnick C, Lindequist U, Screening of Yemeni medicinal plants for antibacterial and cytotoxic activities, Journal of Ethnopharmacology, 2001; 74:173- 179.

[13] Bansemir A, Blume M, Schröder S, Lindequist U, Screening of cultivated seaweeds for antibacterial activity against fish pathogenic bacteria, Aquaculture, 2006; 252:79-84.

[14] Chaouche T, Atik Bekkara F, Haddouchi F, Boucherit Z, Antibacterial activity of different extract of Echium pycnanthum pommel, Journal of Chemical and Pharmaceutical Research, 2012; 4(1):216-220.

[15] Yazdani D, Zainal Abidin M A, Tan YH, Kamaruzaman S, Jaganath IB, Screening of phytochemical from ethnomedicinal plants in Malaysia for use against toxigenic Aspergillus flavus, Journal of Medicinal Plants Research, 2012; 6(42):5464-5468.

[16] Nenaah, Antimicrobial activity of Calotropisprocera Ait (Asclepiadaceae) and isolation of four flavonoid glycosides as the active constituents, World Journal Microbiology Biotechnology, 2013; 29:1255-1262.

[17] Oyeleke SB, Dauda BEN, Boye OA, Antibacterial activity of Ficuscapensis, African Journal of Biotechnology, 2008; 7(10):1414-1417.

[18] Ahameethunisa, Hopper, In vitro antimicrobial activity on clinical microbial strains and antioxidant properties of Artemisia parviflora, Annals of Clinical Microbiology and Antimicrobials, 2012; 11:30.

[19] Hamouda Ali I, Doumandji A, Comparative phytochemical analysis and in vitro antimicrobial activities of the cyanobacterium Spirulina platensis and the green alga Chlorella pyrenoidosa: potential application of bioactive components as an alternative to infectious diseases, Bulletin de l'Institut Scientifique, 2017; 39:41-49.

[20] Ramdan B, Amakran A, Bakrim N, Vannier B, Greche H, Nhiri M, Anti-glycation and radical scavenging activities of hydroalcohol and aqueous extracts of nine species from Lamiaceae family, Journal of Medicinal Plants Studies, 2017; 5(1):331345 .

[21] Nijveldt RJ, van Nood E, van Hoorn DN, Boelens PG, van Norren $\mathrm{K}$, van Leeuwen PAM, Flavonoids: a review of probable mechanisms of action and potential applications, Journal of Clinical Nutrition, 2001; 74:418-425.

[22] Nickavar B, Kamalinejad M, Izadpanah H, In vitro free radical scavenging activity of five Salvia species, Pak. J. Pharm. Sci., 2007; 20(4):291-294.

[23] Wojdyło A, Oszmiański J, Czemerys R, Antioxidant activity and phenolic compounds in 32 selected herbs, Food Chemistry, 2007; 105 (3):940-949.

[24] Hsu B, Coupar IM, NgK, Antioxidant activity of hot water extract from the fruit of the Doum palm, Hyphaenethebaica, Food Chemistry, 2006; 98:317-328.
[25] Zha XQ, Wang JH, Yang XF, Liang H, Zhao LL, Bao SH, Luo JP, Xu YY, Zhou BB, Antioxidant properties of polysaccharide fractions with different molecular mass extracted with hotwater from rice bran, Carbohydrate Polymers, 2009; 78:570575 .

[26] Moran JF, Klucas RV, Grayer RJ, Abian J, Becana M, Complexes of iron with phenoliccompounds from soybean nodules and other legume tissues: Prooxidant and antioxidant properties, Free Radical Biology and Medicine, 1997; 22:861-870.

[27] Bekara A, Ait Hamadouche N, Kahloula K, Sadi N, Aoues A Etude phytochimique et activité antioxydante de l'extrait aqueux de Pimpinella anisum L., Algerian Journal of Natural Products, 2016; 4 (3):299-307.

[28] Lacombe A, Wu VCH, Tyler S, Edwards K, Antimicrobial action of the American cranberry constituents; phenolics, anthocyanins, and organic acids, against Escherichia coli 0157:H7, International Journal of Food Microbiology, 2010; 139:102-107.

[29] Fertout-Mouri N, Latreche A, Mehdadi Z, Bengherraz Z, Activité antibactérienne de quatre extraits de Teucrium polium L. du mont de Tessala (Algérie occidentale), Bulletin de la Société royale des Sciences de Liège, 2016; 85:253-262.

[30] Klančnik A, Piskernik S, Jeršek B, Možina SS, Evaluation of diffusion and dilution methods to determine the antibacterial activity of plant extracts, Journal of Microbiological Methods, 2010; 81:121-126.

[31] Orhan DD, Őzçelik B, Őzgen S, Ergun F, Antibacterial, antifungal and antiviral activities of some flavonoids, Microbiological Research, 2010; 165:496-504.

[32] Yala J-F, Ntsameso-Mve-Mba V, Azzizet Issembe Y, Lepengue NA, Souza A, Évaluation in vitro de l'activité antimicrobienne de l'extrait aqueux d'Eryngium foetidium récolté dans la ville de France ville, Journal of Applied Biosciences, 2016; 103:9886- 9893.

[33] Rodriguez-Vaquero MJ, Alberto MR, Manca de Nadra MC, Infuence of phenolic compounds from wines on the growth of Listeria monocytogenes, Food Control, 2007; 18 (5):587-593.

[34] Boban N, Tonkic M, Modun D, Budimir D, Mudnic I, Davorka Sutlovic D, Punda-Polic V, Boban M, Thermally treated wine retains antibacterial effects to food-born pathogens, Food Control, 2010; 21(8):1161-1165.

[35] Cowan MM, Plant products as antimicrobial agents, Clinical Microbiology Reviews, 1999; 12 (4):564-582.

[36] Benyagoub E, Boulanouar A, Souid Ahmed M, Nebbou N, Bouloufa A, Essai d'évaluation de l'activité antibactérienne de la gomme arabique d'Acacia tortilis(Forssk) contre quelques souches bactériennes pathogènes, Bulletin de la Société Royale des Sciences de Liège, 2016; 85:237-252.

[37] Cushnie TPT, Lamb AJ, Antimicrobial activity of flavonoids, International Journal of Antimicrobial Agents, 2005; 26:343356

[38] Ahmad A, Kaleem M, Ahmed Z, Shafiq H, Therapeutic potential of flavonoids and their mechanism of action against microbial and viral infections-A review, Food Research International, 2015; 77:221-235 